Insertional oncogenesis

\section{Insertional oncogenesis in gene therapy: how much of a risk?}

M Sadelain

Gene Therapy (2004) 11, 569-573. doi:10.1038/sj.gt.3302243

Insertional mutagenesis is an unavoidable consequence of the transposition of genetic material. Whether it involves an integrating virus, a transposable element, a replicationdefective viral vector or plasmid DNA, the ectopic chromosomal integration of DNA is a mutagenic event that may disrupt chromatin or gene structure, thereby altering gene transcription, regulation and/or coding sequences. The gravest concern associated with such mutations is the risk of cell transformation. The acquisition of the malignant phenotype may follow tumor suppressor gene inactivation, typically a recessive event, or oncogene trans-activation, which is likely to exert a dominant effect. The propensity of transposable elements to induce experimental tumors in animals has long been exploited to study the genetic basis of cancer. ${ }^{1-3}$ The earliest analyses of the therapeutic potential of gene transfer have thus recognized the risk of insertional oncogenesis as inherent to the use of integrating vectors. ${ }^{4}$ Yet, there have been extremely few reports documenting insertional oncogenesis in rodent, dog and non-human primate gene transfer studies. ${ }^{5}$ Based on these experimental data, gene therapists and their patients have justifiably assumed that the risk of insertional oncogenesis would be very low.

This reassuring point of view was challenged last year when the firstever case of iatrogenic malignant transformation was reported in a gene therapy patient treated for $X$ linked severe combined immune deficiency (SCID). ${ }^{6}$ The consternation brought upon by this serious adverse event (SAE), which shook the medical community and the concerned regulatory agencies, was compounded, but also mitigated, when a second case was reported 6 months later - in the same trial. ${ }^{7}$ These two SAEs imposed a re- evaluation of the safety concerns associated with gene transfer and of the risk benefit ratio of gene therapy in the context of SCID as well as other diseases. Following a temporary hold on gene therapy trials using retroviral vectors to transduce hematopoietic stem cells, the US Food and Drug Administration and the NIH Recombinant DNA Advisory Committee judiciously concluded that this pair of SAEs did not constitute ground for the cessation of gene therapy investigation. ${ }^{8}$ The interrupted trials were therefore allowed to reopen, pending the case-by-case re-evaluation of the risk/benefit ratio for every study and the appropriate revision of the monitoring plans and of the information provided to prospective patients before obtaining informed consent. $^{8}$

The prompt resumption of these trials is good news for the field, but the need to reassess the safety of the many gene transfer strategies under development remains. Our understanding of the biological mechanisms that determine, in qualitative and quantitative terms, the risk of clonal expansion and transformation, is still fragmentary. However, two recent reports ${ }^{9,10}$ provide valuable insight into the transforming mechanisms that may have operated in the two SCID patients who developed clonal T-cell expansions. Based on these observations, we can begin to identify possible factors that increase the risk that insertional mutagenesis will turn into oncogenesis. While prospective studies are direly needed to analyze and quantify the role of these risk factors, the reports by HaceinBey-Abina et $a l^{9}$ and Dave et $a l^{10}$ provide important clues for taking steps to investigate and reduce the risk of oncogene trans-activation by integrating gene transfer vectors.
Gene therapy of $X$-linked $S C I D$ : landmark success and two SAEs

The two leukemia-like lymphoproliferative disorders arose in patients with X-linked SCID who were treated with a single infusion of autologous CD34+ bone marrow cells transduced with the $\gamma_{c}$ cDNA. Xlinked SCID is a lethal immune deficiency affecting T, B and NK cell development, which is caused by mutations in the gene encoding the common $\gamma$ chain $\left(\gamma_{c}\right)$ shared by the receptors for interleukins 2, 4, 7, 15 and 21. ${ }^{11}$ The dramatic therapeutic response achieved in all but one of 10 treated patients resulted from the restoration of essential features of cellular and humoral immunity ${ }^{12,13}$ arguably the first major success of gene therapy for a congenital monogenic disorder. This landmark achievement was accomplished using an oncoretroviral vector encoding the $\gamma_{c}$ cDNA placed under the transcriptional control of the Moloney murine leukemia virus (Mo-MLV) long terminal repeat (LTR) and a conventional hematopoietic stem cell transduction protocol (entailing cytokine stimulation of purified bone marrow CD34+ cells followed by infection with amphotropic retroviral particles). The transduced cells were transplanted without any host conditioning and it was expected that the cells expressing $\gamma_{c}$ would preferentially expand in the recipient by virtue of the restored responsiveness to multiple cytokines. $^{14}$ In both patients, the leukemias appeared 30-34 months later, in the form of monoclonal or oligoclonal expansion of either $\gamma \delta$ or $\alpha \beta \mathrm{T}$ cells, exceeding levels of $100000 \mathrm{~T}$ lymphocytes per $\mathrm{mm}^{3}$ of blood and causing splenomegaly. The lymphoproliferative syndrome has been successfully treated in both patients, by HLA-matched allogeneic bone marrow transplantation in one instance and by chemotherapy in the other, resulting in complete clinical remissions. ${ }^{9}$

\section{Mechanisms - 1. Oncogene transactivation}

Two recent reports help piece together some of the events that may have led to leukemic transformation. 
A Fischer and his collaborators have performed detailed molecular and genetic analyses, including Southern blot analyses, sequencing, LAMPCR, spectral karyotyping, RNA FISH, immunoscope analyses and cancer susceptibility tests. Their findings directly implicate the vector in the oncogenic process. In both patients, the vector is integrated near an oncogene whose expression is normally repressed in post-thymic $\mathrm{T}$ cells, but is now induced as a consequence of the vector's neighboring integration. The stunner is that, in both instances, the oncogene is one and the same - LMO2. Overexpression of this oncogene has been previously associated with leukemia in patients with acute lymphoblastic leukemia (T-ALL) and in transgenic mice, ${ }^{15-17}$ thus making it highly probable that induction of LMO2 expression is one causal determinant of leukemic tranformation. It remains baffling, however, that out of many potential oncogenes, LMO2 is targeted twice in two independent cases. Indeed, there are many genetic routes to leukemia, and the majority do not involve LMO2., 2,3,18

At first glance, this suggests either that the targeting by the vector's preintegration complex (PIC) is skewed towards the LMO2 locus, or that strong genetic selection is operating to amplify a rare event. The targeting hypothesis requires that cytokine-activated hematopoietic CD34 + progenitors have an accessible LMO2 locus and that it be frequently targeted because of preferential integration at this site or of the high prevalence of a uniquely susceptible lymphoid progenitor population. Both of the latter mechanisms are theoretically plausible, but neither is supported by currently available data: there is no basis for gene-specific targeting by the MoMLV PIC (by tethering to an LMO2associated complex) or evidence for the amplification of a particular $\mathrm{T}$-cell progenitor specifically in $\mathrm{X}$ linked SCID bone marrow. The selection hypothesis also requires that the LMO2 locus be accessible, but only at baseline frequency. This would be followed by powerful mechanisms that preferentially amplify cells overexpressing LMO2. This is consistent with observations in LMO2 transgenic mice that show increased thymocyte proliferation preceding thymic tumor development. ${ }^{16,17}$
However, this does not explain why LMO2-associated leukemias have only occurred in patients with X-linked SCID and not in thousands of mice and $\sim 200$ human patients treated with retrovirally transduced CD34 + cells, ${ }^{5}$ if one assumes that the LMO2 locus was targeted at a similar frequency. This discrepancy may reflect a species difference between humans and mice, the need for an extended preleukemic phase (30 months, never attained in mice), or the engraftment of a lesser dose of transduced cells in the patients in other clinical trials. Alternatively, it may be that LMO2 overexpressing cells proliferate more and hence mutate more in the SCID microenvironment or that preleukemic or leukemic clones are less likely to be eliminated because of the immune deficiency. These hypotheses are not supported by the available preclinical gene transfer studies in SCID mice, but they should be further tested.

\section{Mechanisms - 2. Oncogene cooperativity and the two- hits-in-one hypothesis}

There are other hypotheses on the possible mechanisms involved in leukemic transformation in patients with X-linked SCID. The vector may indeed be doing more than transactivating LMO2. Through its enhancer elements, the integrated vector could activate additional genes located downstream or upstream of LMO2. This possibility should be further examined. Another is that expression of the $\gamma_{c}$ transgene contributes either additively or synergistically to LMO2-associated leukemogenesis. This possibility is supported by the findings of Dave et $a l^{10}$ who examined a large collection of leukemias generated in retrovirusinfected AKXD mice. Neonatal mice undergoing active retroviral infection are susceptible to insertional oncogenesis caused by oncogene transactivation and/or tumor suppressor gene disruption., ${ }^{2,3}$ The authors queried the tumor database established by $\mathrm{N}$ Copeland and $\mathrm{N}$ Jenkins (http://RTCGD.ncifcrf.gov), in search for LMO2 integration sites. Starting from a series of 3000 proviral integration sites, they identified two leukemias in which the LMO2 locus was a target for retroviral integration, leading to LMO2 overexpression. ${ }^{10}$ Remarkably, one of the two clones bore a proviral integration near the $\gamma_{c}$ (or IL2RG) locus - in itself an equally rare occurrence which also lead to dysregulated $\gamma_{c}$ expression. This very unlikely event (with an estimated probability of $10^{-6}$ in a panel of 600 tumors, Dave et $a l^{10}$ ) suggests some functional complementarity, akin to oncogene cooperativity, between LMO2 and $\gamma_{c}$.

This strong genetic evidence is neither corroborated nor disavowed by the functional data currently available. $\gamma_{c}$ itself is not known to be an oncogene, and no tumors have been reported to date in mice overexpressing $\gamma_{c}{ }^{5}$ It was argued earlier that, because $\gamma_{c}$ expression is con stitutive in many hematopoietic cell types, ${ }^{11}$ LTR-driven expression would not be particularly disruptive. Indeed, the levels of $\gamma_{c}$ expression found in the two patients' leukemic clones are in the physiologic range. ${ }^{9}$ It was also thought that the functional consequences of expressing $\gamma_{c}$ under the transcriptional control of a nonspecific retroviral enhancer/promoter would be restrained by the association with the other chains constituting the interleukin receptors (which were presumed to remain appropriately regulated in the SCID host). These assumptions warrant additional investigation. It is noteworthy, for example, that $\gamma_{c}$ expression is physiologically downregulated following cytokine activation, ${ }^{19}$ while the Mo-MLV LTR, conversely, increases its transcriptional activity following T-cell activation. ${ }^{20}$ All considered, the twohits-in-one hypothesis needs to be vigorously pursued in direct animal experimentation.

\section{An early assessment of risk factors for insertional oncogenesis}

Based on the recent reports by Hacein-Bey-Abina et al and Dave et al, one can begin to identify risk factors favoring insertional oncogenesis, at least in the case of recombinant oncoretroviral vectors (Table 1). There is presently less information on integration site selection for other vector categories, including those derived from HIV-1, AAV, herpes 
Table 1 Potential risk factors for insertional oncogenesis

Number of hits

Integration site selection

Nature of mutagenesis Ubiquitous expression Nature of target cell

Cell dose

Host immune competence

Host genetic background Transgene function
Less is better: 1-2 vector copies per cell safest

Proximity to genes; bias towards promoter regions; effect of cell cycle status and cytokines on repertoire of accessible loci

Potential for generating fusion genes; range over which chromatin structure/transcription can be altered Nonspecific transcriptional activity in all cells at all times undesirable if unnecessary

Higher proliferative potential of stem and progenitor cells may pose a greater risk

More engrafted cells implies more integration sites, including potentially hazardous sites

Defective immune surveillance may permit progression of transformation; imbalanced cytokine milieu

may increase homeostatic proliferation or clonal expansion

May affect vector integration, vector expression, cancer susceptibility

See Table 2

Table 2 Risk stratification of constitutively expressed transgenes

Level 1

Level 2

Level 3

Unknown
No known toxicity over a wide range of expression levels; absence of effect on cell survival or cell proliferation Possible toxicity at aberrant levels of expression; potential effect on cell survival or cell proliferation under extreme conditions; cotransforming function under conditions of cooperativity Known to cause tumors in animal models Incomplete elucidation of transgene product functions; insufficient experimental gene transfer data in relevant disease models virus, adenovirus, SV40, as well as plasmid DNA. ${ }^{21}$ The gene therapy literature ${ }^{5}$ and the recent findings with $\gamma_{c}{ }^{9,10}$ also point to the importance of the transgene product itself in causing or facilitating transformation. In the case of murine insertional oncogenesis caused by the integration of a replication-defective oncoretroviral vector near the Evi-1 oncogene, ${ }^{22}$ the transgene, encoding a partially inactivated form of the human LNGFR under the transcriptional control of the vector's LTR, was expressed in the myeloid leukemia. It remains controversial whether its expression may have contributed to clonal expansion or transformation in that instance. ${ }^{22,23}$ Altogether, these findings suggest a classification of transgenes in terms of their potential to contribute to malignancy when they are constitutively expressed (Table 2). Most importantly, all of these risk factors, whether known or hypothetical, can be studied and quantified in prospective animal studies.

\section{Remedies against insertional oncogenesis}

There are several steps one can take to reduce the probability of oncogene activation in the absence of having control over integration site selection. The first and most obvious is to introduce few vector copies per cell and thus reduce the number of potential hits. In the cases where high level expression of the transgene product is required, such as for the severe hemoglobinopathies, it is therefore essential to conceive vectors that provide the highest possible level of $\beta$ or $\gamma$ globin expression to achieve maximum therapeutic benefit with the lowest possible vector copy number. ${ }^{24}$ The other steps aim to curtail the vector's ability to transactivate neighboring genes. ${ }^{21}$ This can be achieved by improving vector design, relying on mechanisms such as LTR deletion, tissue-specific expression, embedded transcription and insulation (the three latter mechanisms can be applied to gene transfer vectors other than recombinant retroviruses). The LTR deletion aims to inactivate the $3^{\prime}$ LTR, reduce the number of active enhancer/promoters in the vector and eliminate a nonspecific enhancer (Figure 1a). The confinement of transcriptional initiation, attenuation and polyadenylation within vector boundaries aims to minimize transcription of flanking chromosomal sequence and preclude the generation of $\mathrm{fu}-$ sion gene products (Figure $1 \mathrm{~b}$ ). Tissue-specific expression aims to restrict transcription to the relevant cells only and avoid transcription in hematopoietic progenitors (Figure 1c). Finally, insulators and other elements with enhancer blocking activity may help to prevent onco- gene transactivation (Figure 1d). The vogue for insulators should be nonetheless tempered and needs to be carefully investigated, as these elements may help reduce trans-activation of adjacent genes ${ }^{21}$ but might also act as mutagens by disrupting gene regulation or by altering DNA methylation. ${ }^{25,26}$ All in all, a number of modifications to vector design can be made to reduce the risk of insertional oncogenesis. These need to be experimentally validated.

\section{How much of a risk?}

Simply put, the central question is what is the probability for any given gene therapy strategy to cause secondary malignancy. A quantitative answer to this question will permit rational evaluation of the risk/benefit ratio for the concerned patient population. Until recently, the probability of tumor formation seemed to be very low, based on rather extensive preclinical research. ${ }^{5}$ The two major SAEs incurred in patients with $\mathrm{X}$-linked SCID challenge this view, but their occurrence, as troubling as it may be, does not call into question the cogency of gene transfer-based medicine. As attested by the findings of Hacein-Bey-Abina et al and Dave et al, the path to leukemic transformation, which requires more than a single genetic hit, is by no means evident in these two patients, and is 

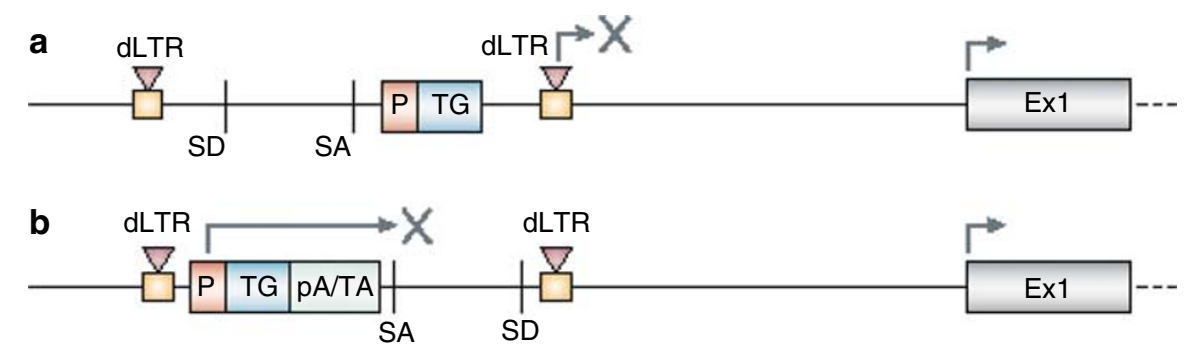

C
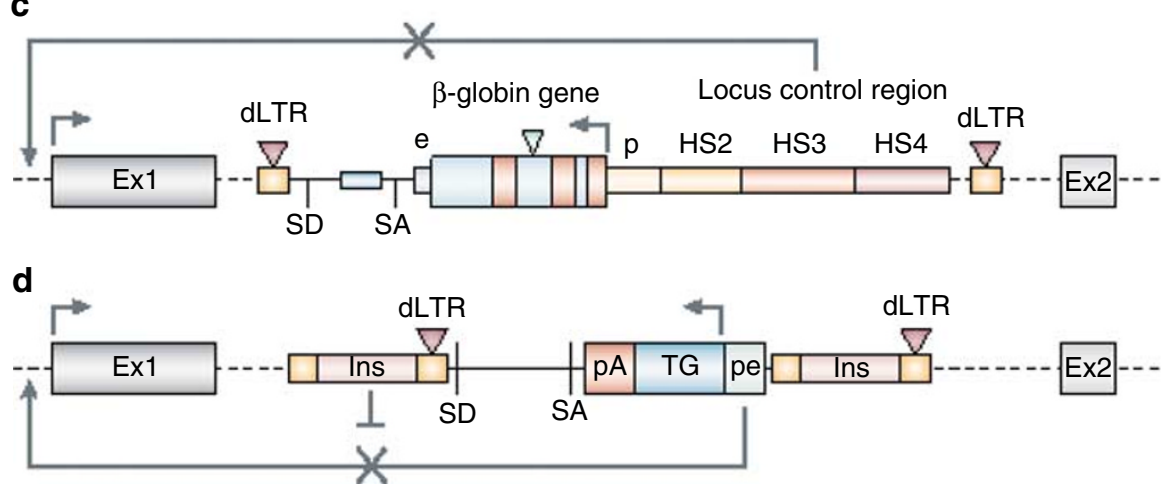

Figure 1 Vector modifications to reduce the risk of proto-oncogene trans-activation. (Reprinted by permission from Nat Rev Cancer (Vol 3, no. 7, pp 477488, copyright (2003) Macmillan Magazines Ltd.; http://www.nature.com/reviews) (a) Inactivation of the LTRs by U3 deletion to reduce the number of active enhancer/promoters in the vector and eliminate a nonspecific enhancer. (b) Abrogation of transcriptional read-through by transcriptional attenuation and strong polyadenylation to confine transcription within vector boundaries. (c) Lineage-restricted, differentiation stage-restricted transcription. For example, a vector expressing only in maturing erythroblasts would not be transcriptionally active in $T$ cells or hematopoietic progenitors. (d) Insulation by flanking the vector with insulator elements (Ins) or other elements with enhancer blocking activity. In (a) and (b), the vector is integrated upstream of the oncogene's first exon, and, in (c) and (d), in the first intron of the oncogene, as found in patients 5 and 4, respectively. ${ }^{9}$ SD: splice donor; SA: splice acceptor; dLTR: U3-deleted LTR; P: internal promoter; TG: transgene; $p$ A: polyadenylation signal; e; enhancer; Ins: insulator.

Table 3 Recommendations for murine gene transfer studies to optimize the yield of relevant information ${ }^{\mathrm{a}}$

- Hematological analyses and necropsies should be systematically performed in long-term hematopoietic chimeras. It would be reasonable to request that preclinical studies used to support proposed gene therapy trials perform a set of standard hematological analyses.

- A proportion of treated animals should be maintained for at least 1 year. The classic 4-month follow-up is minimally informative with respect to assessing oncogenicity.

- Long-term follow-up should be highly valued by reviewers and editors; papers should clearly indicate the number of mice and duration of follow-up (these parameters are often difficult to obtain).

- Therapeutic genes should be tested in relevant disease models.

- Preclinical animal studies should appropriately mimic the human trials. The experiments should attempt to use animals of an appropriate age, relevant gene transfer efficiencies, transduction conditions, cell doses and conditioning regimens.

- Novel transgenes should be tested in transgenic mice as well as in hematopoietic chimeras. The effect of increased expression of the $\gamma_{c}$ chain in the development of the T-cell leukemias is not known. More studies are needed to determine the need for tighter regulation of $\gamma_{c}$ expression (temporal regulation, level).

- Integration sites should be monitored, at least in the event of a myeloproliferative or lymphoproliferative disorder. Now that the mouse genome is publicly available, the determination of insertion location should become part of the experimental plan in selection studies. At the least, DNA should be stored from all animals at the time of killing so that later analyses can be performed in the setting of unexplained adverse events.

- The integration sites should be related to target cell type, transduction conditions, clonal expansion, duration of transgene expression and toxicities.

- Investigators in the field of gene therapy should share and centralize the information with the goal of establishing a large database of integration sites to assess the frequency of retroviral integration sites and their risk.

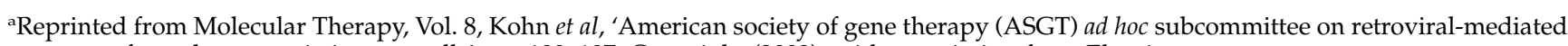
gene transfer to hematopoietic stem cells', pp 180-187, Copyright (2003), with permission from Elsevier.

further muddled by the extraordinary two-time targeting of the same locus. Far from suggesting a generalizable phenomenon, the SAEs associated with $\gamma_{c}$ therapy for X-linked SCID may represent a unique situation.
Nonetheless, this precedent mandates heightened monitoring in animal studies and clinical trials. An ad hoc subcommittee established by ASGT recently promulgated a series of recommendations designed to assess the frequency of insertional oncogenesis in rodent studies..$^{5}$ Of particular importance is the need to perform experiments under conditions that closely mimic the clinical setting and to carefully monitor the hematology and general health status in long-term hematopoietic chi- 
meras (Table 3). This will require a long-term effort entailing the systematic study of all oncogenic risk factors (Table 1) and the specific risk of each transgene (Table 2).

As for remedies, it will be imperative to perform experimental studies and clinical trials with delivery of a low vector copy number. Evidently, the integration of multiple proviral copies per cell following RCR infection or deliberate high vector copy transfer is poised to increase the risk of transformation. The crucial questions are how to control the gene delivery rate effectively, so as to integrate only 1-2 vector copies per cell, and how to reduce the probability of trans-activating oncogenes, especially in stem and progenitor cells. Altogether, there are several improvements in vector design that can be developed to pre-empt gene therapy-related secondary malignancies. The challenge is to convince the medical community and the public that these auspicious safeguards work.

Dr M Sadelain is at Memorial Sloan-Kettering Cancer Center, New York, NY, USA. e-mail: m-sadelain@ski.mskcc.org

1 Nusse $\mathrm{R}$ et al. Mode of proviral activation of a putative mammary oncogene (int-1) on mouse chromosome 15. Nature 1984; 307: 131-136.

2 Mikkers $\mathrm{H}$ et al. High-throughput retroviral tagging to identify components of specific signaling pathways in cancer. Nat Genet 2002; 32: 153-159.

3 Suzuki $\mathrm{T}$ et al. New genes involved in cancer identified by retroviral tagging. Nat Genet 2002; 32: 166-174.

4 Friedmana T. The future for gene therapy - a reevaluation. Ann NY Acad Sci 1976; 265: 141-152.
$5 \mathrm{Kohn} \mathrm{DB}$, et al. American Society of Gene Therapy (ASGT) ad hoc subcommittee on retroviral-mediated gene transfer to hematopoietic stem cells. Mol Ther 2003; 8: 180-187.

6 Hacein-Bey-Abina $S$ et al. A serious adverse event after successful gene therapy for X-linked severe combined immunodeficiency. N Engl J Med 2003; 348: 255-256.

7 Marshall E. Gene therapy. Second child in French trial is found to have leukemia. Science 2003; 299: 320.

8 Friedmann T. Gene therapy's new era: a balance of unequivocal benefit and unequivocal harm. Mol Ther 2003; 8: 5-7.

9 Hacein-Bey-Abina $\mathrm{S}$ et al. LMO2associated clonal $\mathrm{T}$ cell proliferation in two patients after gene therapy for SCID-X1. Science 2003; 302: 415-419.

10 Dave UP, Jenkins NA, Copeland NG. Gene therapy insertional mutagenesis insights. Science 2004; 303 (5656): 333.

11 Leonard WJ. Cytokines and immunodeficiency diseases. Nat Rev Immunol 2001; 1: 200-208.

12 Cavazzana-Calvo $\mathrm{M}$ et al. Gene therapy of human severe combined immunodeficiency (SCID)-X1 disease. Science 2000; 288: 669-672.

13 Hacein-Bey-Abina $S$ et al. Sustained correction of X-linked severe combined immunodeficiency by ex vivo gene therapy. N Engl J Med 2002; 346: 1185-1193.

14 Lantz O, Grandjean I, Matzinger P, Di Santo JP. Gamma chain required for naive CD4+ $\mathrm{T}$ cell survival but not for antigen proliferation. Nat Immunol 2000; 1: 54-58.

15 Royer-Pokora B, Loos U, Ludwig WD. TG-2, a new gene encoding a cysteinerich protein with the LIM motif, is overexpressed in acute T-cell leukaemia with the $t(11 ; 14)(\mathrm{p} 13 ; \mathrm{q} 11)$. Oncogene 1991; 6: 1887-1893.

16 Larson RC et al. The oncogenic LIM protein Rbtn2 causes thymic developmental aberrations that precede malignancy in transgenic mice. Oncogene 1995; 11: 853-862.

17 Neale GA, Rehg JE, Goorha RM. Ectopic expression of rhombotin-2 causes selective expansion of CD4-CD8lymphocytes in the thymus and T-cell tumors in transgenic mice. Blood 1995; 86: 3060-3071.

18 Ferrando AA et al. Gene expression signatures define novel oncogenic pathways in $\mathrm{T}$ cell acute lymphoblastic leukemia. Cancer Cell 2002; 1: 75-87.

$19 \mathrm{Li}$ XC et al. IL-15 and IL-2: a matter of life and death for $\mathrm{T}$ cells in vivo. Nat Med 2001; 7: 114-118.

20 Pollok KE et al. Costimulation of transduced $\mathrm{T}$ lymphocytes via $\mathrm{T}$ cell receptor-CD3 complex and CD28 leads to increased transcription of integrated retrovirus. Hum Gene Ther 1999; 10 2221-2236

21 Kohn DB, Sadelain M, Glorioso JC. Occurrence of leukaemia following gene therapy of X-linked SCID. Nat Rev Cancer 2003; 3: 477-488.

$22 \mathrm{Li} \mathrm{Z}$ et al. Murine leukemia induced by retroviral gene marking. Science 2002; 19: 296-497.

23 Bonini C et al. Safety of retroviral gene marking with a truncated NGF receptor. Nat Med 2003; 9: 367-369.

24 Rivella S, Lisowski L, Sadelain M. Globin gene transfer: a paradigm for transgene regulation and vector safety. Gene Ther Reg 2003; 2: 149-175.

25 Gerasimova TI, Corces VG. Chromatin insulators and boundaries: effects on transcription and nuclear organization. Annu Rev Genet 2001; 35: 193-208.

26 Rivella $\mathrm{S}$ et al. The cHS4 insulator increases the probability of retroviral expression at random chromosomal integration sites. J Virol 2000; 74: 4679-4687. 